\title{
Big Five Personality Detection Using Deep Convolutional Neural Networks
}

\author{
Waiel Tinwala \\ Dept. of Computer Science and Engineering \\ Delhi Technological University \\ Delhi, India \\ Waieltinwala_2k18co399@dtu.ac.in
}

\author{
Shristi Rauniyar \\ Dept. of Computer Science and Engineering \\ Delhi Technological University \\ Delhi, India \\ Shristirauniyar_2k18co343@dtu.ac.in
}

\begin{abstract}
Personality is the most critical feature that tells us about an individual. It is the collection of the individual's thoughts, opinions, emotions and more. Personality detection is an emerging field in research and Deep Learning models have only recently started being developed. There is a need for a larger dataset that is unbiased as the current dataset that is used is in the form of questionnaires that the individuals themselves answer, hence increasing the chance of unconscious bias. We have used the famous stream-of-consciousness essays collated by James Pennbaker and Laura King. We have used the Big Five Model often known as the five-factor model or OCEAN model. Document-level feature extraction has been performed using Google's word2vec embeddings and Mairesse features. The processed data has been fed into a deep convolutional network and a binary classifier has been used to classify the presence or absence of the personality trait. Holdout method has been used to evaluate the model, and the F1 score has been used as the performance metric.
\end{abstract}

Keywords-Personality Detection, Big Five model, Fivefactor Model, OCEAN, Deep Convolutional Neural Network, Mairesse Features, stream-of-consciousness

\section{INTRODUCTION}

Personality is defined as nature, characteristics or qualities that form an individual's character. It is the combination of the individual's thought process, opinions, emotions, fears, goals, and aspirations.

The personality of the individual is the factor that determines how an individual is going to be with their family and friends, what kind of career they will opt for, how they will perform at work and exist in the global community. When individuals are well-versed with their personality, they know about their strengths and weaknesses which will help in self-development.

Some widely used theories for classifying personality traits are the Big Five model, Myers-Briggs Type Indicator, Keirsey Temperament Sorter, Three-trait personality model and Eysenck Personality Questionnaire [1] .

In this paper, we are going to classify personalities on the Big Five classification. This classification remains relatively uniform throughout one's lifetime and is the most widely accepted personality classification theory adapted by psychologists and researchers worldwide.

The Big Five model classifies personalities under five broad umbrella terms that encompass more labels. The five personality categories are described below:

- Conscientiousness (CON): This trait describes the individual's ability to control impulses for goaloriented tasks. It describes the ability of the individual to be organized, responsible, hard-working and adhering to the rules and regulations. If this trait is dominant in an individual, the individual is competent, self-disciplined and driven to achieve their goals. If this trait is not dominant, then it tells us that the individual is careless, procrastinates and is disorganized.

- Agreeableness (AGR): This trait is all about how an individual treats others in relationships. Individuals dominantly having this trait are perceived as straightforward, modest, sympathetic, and forgiving. On the other hand, individuals having a low measure of this trait are perceived as demanding, insulting, stubborn and unsympathetic.

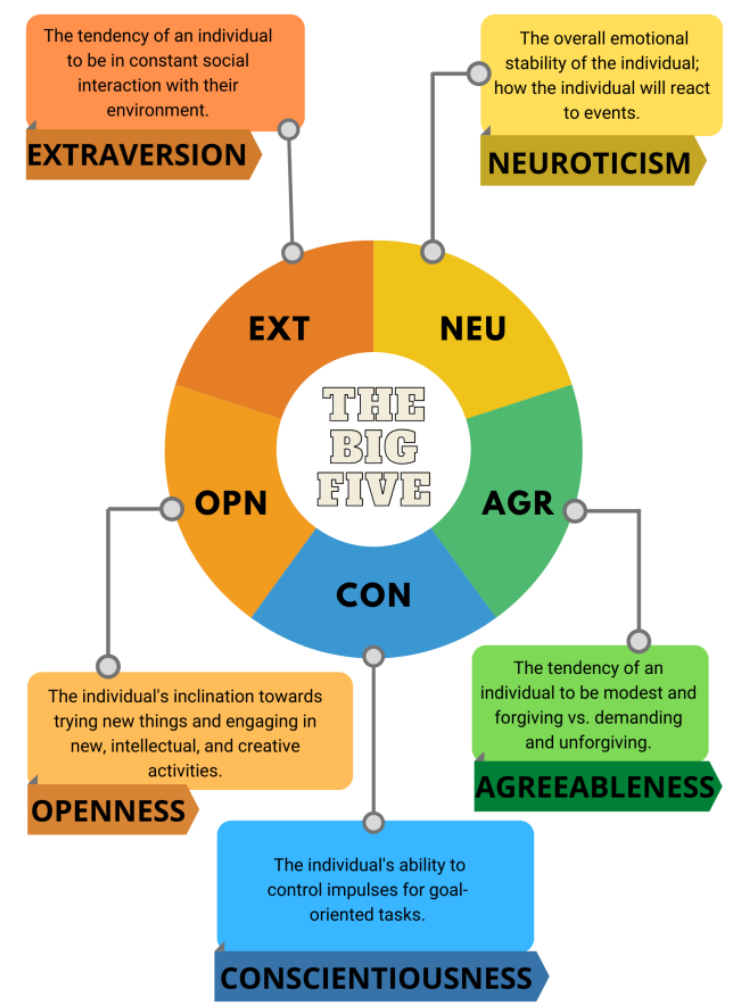

Fig. 1. The Big Five Model

- Extraversion (EXT): This trait describes the tendency of an individual to be in constant social interaction and be interacting with their environment. Having a high measure of extraversion means the individual is very outgoing and enjoys being the centre of attention. Having a low measure of extraversion means that the individual is reserved in nature, prefers solitude over social interaction and does not want to be the centre of attention.

- Openness (OPN): This trait reflects an individual's inclination towards trying new things and engaging in new, intellectual and creative activities. An individual having a high measure of openness means they are creative and think outside of the box. A low measure 
of this trait indicates that the individual prefers routine and traditional ways over new methods.

- Neuroticism (NEU): This trait tells us about the overall emotional stability of the individual. It tells us how the individual is likely to interact and react to events. Having a high measure of this trait means that the individual is likely anxious, easily irritable and experiences mood shifts. An individual with a low measure of this trait is calm, confident and resilient.

The rest of this paper is organized as: related works, methodology, experimental results, conclusion, future work, acknowledgment and references.

\section{RELATED WORKS}

The first Personality Test ever conducted is commonly known as Woodworth Psychoneurotic Inventory and was developed during World War I. The U.S. military used it for screening army recruits. The screening was done for Post Traumatic Stress Disorder (PTSD). The Process Communication Model (PCM) was widely used. PCM was built by Taibi Kahler and was used for the selection of astronauts [1] .

The baseline methods of detecting personality are text, audio and visual cues. When using textual data, data preprocessing is a crucial step that directly affects the results. Generally, the textual features are extracted from the raw data and fed as input into Machine Learning models like Support Vector Machines (SVMs), Naïve Bayes Classifier, etc. The word embeddings are represented as vectors (Word2Vec, GloVe, etc.) and later more techniques are applied to yield results.

Kasula Chaithanya Pramodh et al. in 2016 [2] used the stream-of-consciousness and MyPersonality dataset. The MyPersonality dataset is a compilation of 250 users updating around 10,000 Facebook Status Updates. They have used Natural Language Toolkit for their model and their F1-scores are $0.665,0.632,0.625,0.624$ and 0.637 for OPN, CON, EXT, AGR and NEU traits respectively.

Carles Venture et al. [3] have used Convolutional Neural Networks in combination with Action Unit Recognition Systems and face detection to classify personality as per the Big Five model. They have used the First Impressions dataset that comprises of around 10,000 videos of various individuals.

Gokul K et al. [4] in 2018 have applied Bayes-Net classifier to classify personality as extrovert or introvert. They have used self-recorded audio samples as their dataset. Auditory Nerve Modeling that comprises of Voice Activity Detection in combination with Attribute Selection for dimensionality reduction was applied and they achieved an accuracy of $88.3 \%$.

Bojan Simoski et al. [5] have developed a novel technique called Social Contagion Model for classifying personality on the Big Five Model. They used the responses of 25 individuals to the Big Five Questionnaire as their dataset.

Abir Abyaa et al. in 2018 [6] have used the StudentLife dataset that comprises of data from a group of 48 students. They have made use of supervised learning algorithms for the purpose of classification of personality as per the Big
Five model. The used Support Vector Machines, Random forests, Logistic Regression, C4.5 Decision Tree and knearest neighbours algorithm.

Willy et al. in 2019 [7] have implemented C4.5 Decision Tree for classification of personality based on the Big Five Model. They have used the Twitter API to obtain the dataset that is roughly around 110 million tweets every day. They were able to obtain an accuracy of $64.30 \%$.

In 2019, Tao Hong et al. [8] conducted a research to classify sentiment and detect personality. They have used the MDSTC dataset (comprises of speech, facial expressions and Galvanic Skin Response) and applied Deep Neural Network to classify personality. They achieved better results on performance metrics as compared to the state-of-the-art models.

Imanuel Buhapoda Drexel [9] has used Gaussian Naïve Bayes classifier to detect personality on the basis of Big Five Model. They have applied Word2Vec embeddings as well as FastText word embedding and used k-fold cross validation $(\mathrm{k}=5)$ for cross validation.

Songcheng Gao et al. in 2020 [10] have used a MultiView Learning Approach that also includes a Multi-Task approach with StudentLife dataset that they obtains by developing a mobile application. They collected data from a group of 183 individuals from two different universities. The data collection is in the form of a questionnaire that one has to fill out on the mobile application. They have used mean absolute error and root mean squared error as the performance measure.

Marwa S. Salem et al. [11] have applied Multinomial Naïve Bayes, K-Nearest Neighbour, Support Vector Machine and Decision Trees on Egyptian Twitter Users Dataset to classify personality. According to their research, K-Nearest Neighbor was the best algorithm for Openness, Extraversion, Agreeableness and Neuroticism whereas Decision Tree performed best for Conscientiousness.

Kamal El - Demerdash et al. [12] have used Universal Language Model Fine-Tuning for personality trait detection on the Big Five model. They have applied this Model on the stream-of-consciousness dataset and they have managed to achieve around $1 \%$ better accuracy than most state-of-the-art models.

In 2017, a state-of-the-art research was performed by Navonil Majumder et al. in 2017 [13] where the researchers used a Deep Convolutional Neural Network to classify personality traits basis on the Big Five model. The model is coded in Python 2.7 and uses Google's word2vec embeddings and Mairesse features. This research was improved by Md. Abdur Rahman et al. in 2019 [14], where the researchers applied different activation functions like Tanh, ReLU and leaky ReLU and observed significant improvement on the results. Even this was coded in Python 2.7 .

We have coded the same model in Python 3.8 since Python 3.8 since python 2.7 is no longer supported (as of January 1, 2020). The methodology is explained in detail in the following section. 


\section{METHODOLOGY}

A Deep Convolutional Neural Network $(\mathrm{CNN})$ has been trained to perform Personality Classification. This approach classifies five different personality traits: CON, AGR, EXT, OPN and NEU using the same network architecture. The methodology is described in detail in the following sections:

\section{A. Dataset}

The stream-of-consciousness essay dataset has been used. It is an assortment of 2,468 essays that are composed by people in a controlled environment. The writers also marked their personality traits themselves. The dataset was compiled by James Pennebaker and Laura King.

\section{B. Data Preprocessing}

Since the dataset contains of noise, we have performed cleaning by expanding abbreviations, removing unnecessary symbols and converting the text to lower-case. After performing cleaning, sentence-splitting was performed. The unique words are complied into a list that we have termed as vocabulary.

\section{Deep CNN Architecture}

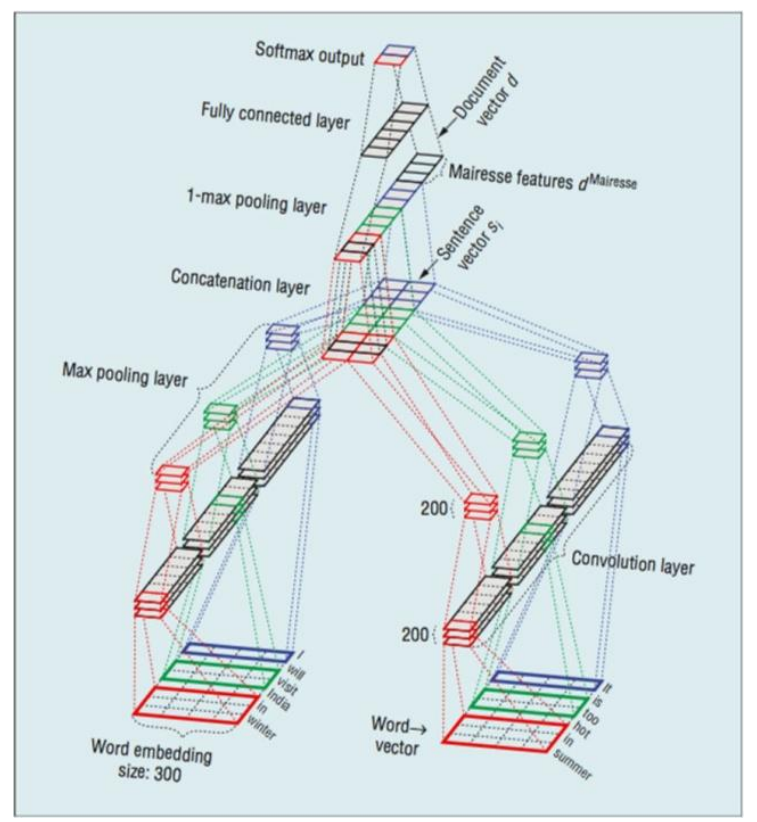

Fig. 2. Network Architecture [13] below:

The different layers are shown in Figure 1 and described

1) First layer-Word vectorization using Word2Vecs: Word-level feature extraction was performed using Google's Word2Vec Word embeddings library and the unknown words not found in Word2Vec were taken care of using uniform distribution. Equation (1) and Fig. 2 explain the Uniform Distribution Function.

$$
F(x)=\left\{\begin{array}{rc}
0, & x<a \\
\frac{x-a}{b-a}, & a \leq x \leq b \\
1, & x>b
\end{array}\right.
$$

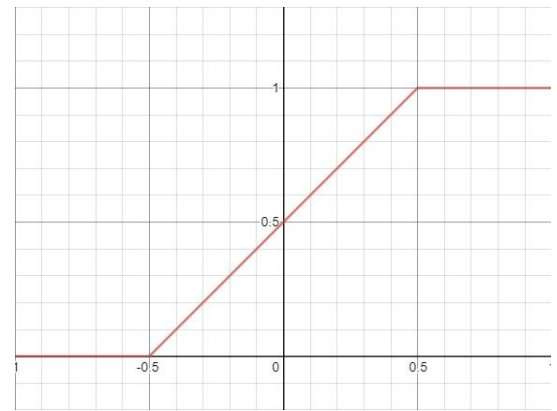

Fig. 3. Uniform Distribution Function

2) Second layer-Convolution: Convolutional filters along with max pooling were applied to the document in order to extract n-gram features from each sentence. The obtained feature vectors were concatenated into a sentence vectors.

3) Third layer-1-max pooling: The sentence vectors from Layer 3 were concatenated into document vectors. The dimension of a sentence vector is the multiple of n-grams. The document is considered only if it belongs to at least one feature.

4) Fourth layer-Concatenation: Mairesse features were concatenated to the document features obtained in Layer 3 were added to the document vector. Mairesse features are a set of document-level features such as Linguistic Inquiry and Word Count Features, utterance-type features, Medical Research Council features, and prosodic features.

5) Fifth layer-Activation: Sigmoid, Tanh and ReLU functions has been used as activation function. Sigmoid function is explained in (2) and Fig. 4. Tanh function is explained in (3) and Fig. 5. ReLU function is explained in (4) and Fig. 6.

$$
S(x)=\frac{1}{1+e^{-x}}
$$

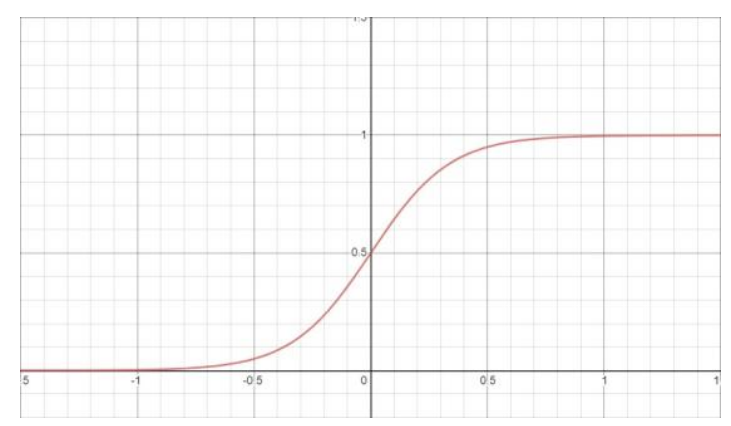

Fig. 4. Sigmoid Function

$$
\operatorname{Tanh}(x)=\frac{\sinh (x)}{\cosh (x)}=\frac{e^{x}-e^{-x}}{e^{x}+e^{-x}}
$$

6) Sixth layer-Classification: The sixth and last layer is a binary classifier that classifies if the given personality trait is present or not by using softmax activation function (5) which is the same as sigmoid function for binary classifiers.

$$
\sigma(\vec{z})_{i}=\frac{e^{z_{i}}}{\sum_{j=1}^{K} e^{z_{i}}}
$$




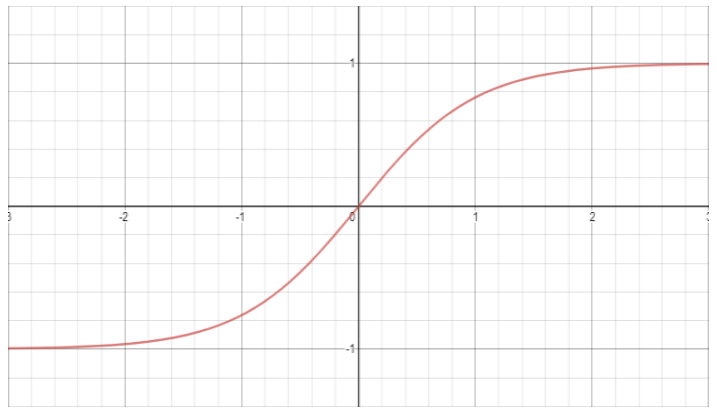

Fig. 5. Tanh Function

$$
F(x)= \begin{cases}0, & x<0 \\ x, & x \geq 0\end{cases}
$$

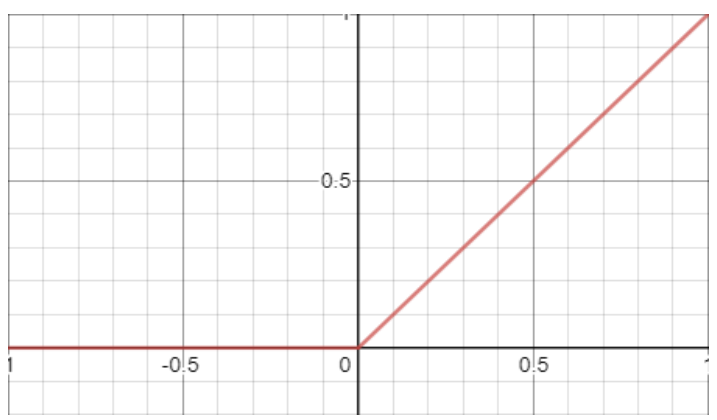

Fig. 6. ReLU Function

\section{EXPERIMENTAL RESULTS}

The constructed dataset is trained and evaluated using hold-out technique. The performance measure that is used is F1 score. It is the harmonic mean of Precision and Recall. True Positive (TP), False Positive (FP), True Negative (TN), False Negative (FN) are the measures used to calculate Precision and Recall. F1-score is given by (8):

$$
\begin{gathered}
\text { Precision }=\frac{T P}{T P+F P} \\
\text { Recall }=\frac{T P}{T P+F N} \\
F 1-\text { score }=\frac{2 * \text { Precision } * \text { Recall }}{\text { Precision }+ \text { Recall }}
\end{gathered}
$$

The results that we have obtained are described in Fig. 7.

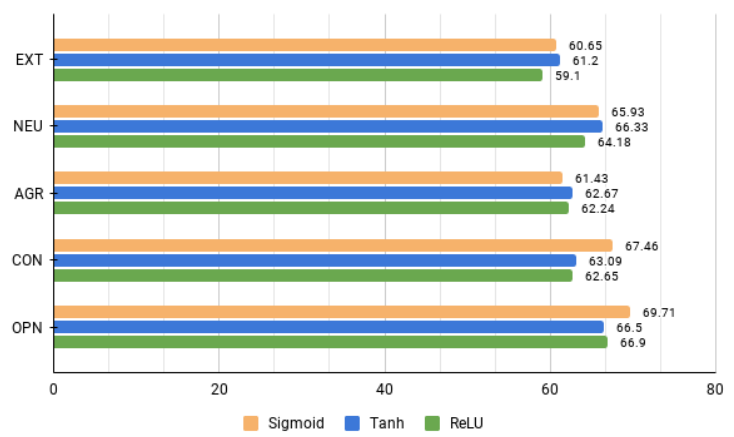

Fig. 7. F1-scores when applying various activation functions

We can observe that Tanh function performs best for traits Extraversion, Neuroticism and Agreeableness with F1scores $61.2 \%, 66.33 \%$ and $62.67 \%$ respectively. Sigmoid performs best for Openness and Conscientiousness with F1scores of $69.71 \%$ and $67.46 \%$ respectively.

\section{COMPARATIVE ANALYSIS}

Activation functions are used in neural networks as outputs for the nodes in the layers. They are used to add nonlinearity to the network. We have used 3 different activation functions for our model namely: Sigmoid, Tanh, and ReLU.

The state-of-the-art model has been trained for 6 epochs. The model was evaluated using $\mathrm{k}$-fold cross-validation $(\mathrm{k}=10)$. Due to GPU and CPU constraints, we have trained our model for 50 epochs and evaluated our model using hold-out cross-validation technique with 9:1 as the train-test ratio.

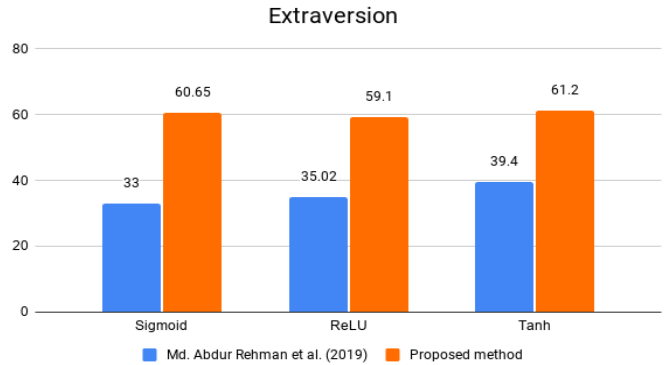

Fig. 8. F1-score comparison of Personality Trait: Extraversion

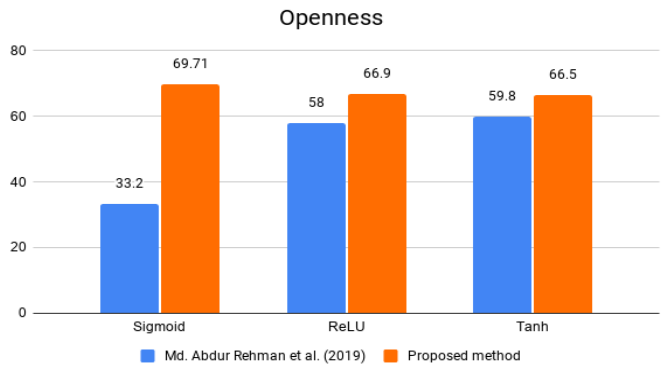

Fig. 9. F1-score comparison of Personality Trait: Openness

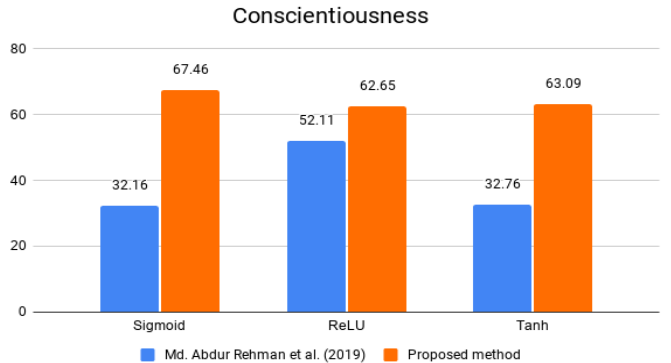

Fig. 10. F1-score comparison of Personality Trait: Conscientiousness

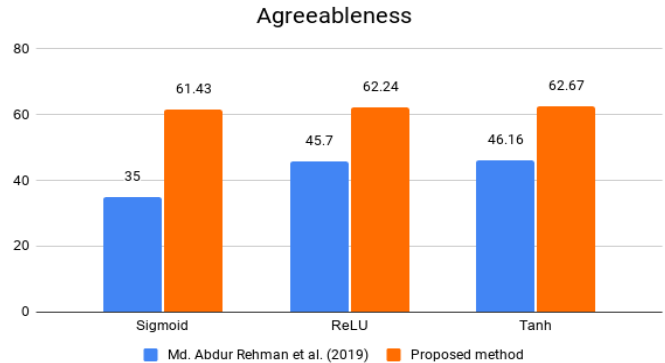

Fig. 11. F1-score comparison of Personality Trait: Agreeableness 


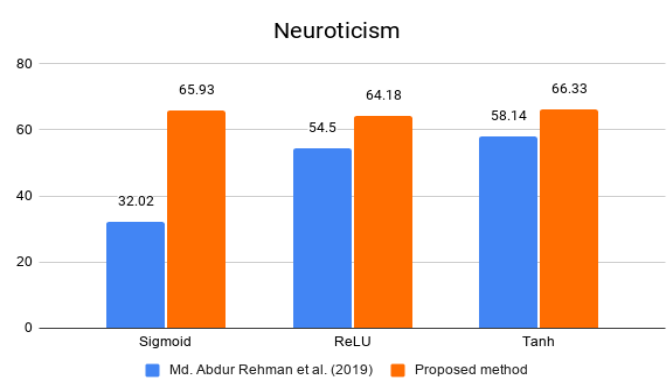

Fig. 12. F1-score comparison of Personality Trait: Neuroticism

Sigmoid and Tanh are non-linear activation functions whereas ReLU is a linear function. We have trained our model using these three activations and compared our results. The comparison for each of the five traits can be seen in Fig. $8,9,10,11$ and 12 . We observe that the non-linear functions i.e.; Sigmoid and Tanh performed better than the linear activation ReLU in all cases. We also observe that our model performs better than the state-of-the-art model.

\section{CONCLUSION}

We have been able to successfully code this model in Python 3.8 and have been able to optimize the previous code structure as well. We have been able to achieve better performance measures than previous state-of-the-art methods as can be observed in Fig. 5. The proposed model had used $\mathrm{k}$-fold cross-validation technique $(\mathrm{k}=10)$ whereas we have used hold-out method because of limited resources and yet have achieved better results.

TABLE I. F1-SCORES COMPARISON TABLE

\begin{tabular}{|l|c|c|c|}
\hline $\begin{array}{l}\text { Personality } \\
\text { Traits }\end{array}$ & $\begin{array}{c}\text { Activation } \\
\text { Function }\end{array}$ & $\begin{array}{c}\text { Md. Abdur Rehman } \\
\text { et al. (2019) }\end{array}$ & $\begin{array}{c}\text { Proposed } \\
\text { method }\end{array}$ \\
\hline \multirow{4}{*}{ EXT } & Sigmoid & 33.00 & 60.65 \\
\cline { 2 - 4 } & ReLU & 35.02 & 59.10 \\
\cline { 2 - 4 } & Tanh & 39.40 & 61.20 \\
\hline \multirow{5}{*}{ NEU } & Sigmoid & 32.02 & 65.93 \\
\cline { 2 - 4 } & ReLU & 54.50 & 64.18 \\
\cline { 2 - 4 } & Tanh & 58.14 & 66.33 \\
\hline \multirow{5}{*}{ AGR } & Sigmoid & 35.00 & 61.43 \\
\cline { 2 - 4 } & ReLU & 45.70 & 62.24 \\
\cline { 2 - 4 } & Tanh & 46.16 & 62.67 \\
\hline \multirow{5}{*}{ OPN } & Sigmoid & 32.16 & 67.46 \\
\cline { 2 - 4 } & ReLU & 52.11 & 62.65 \\
\cline { 2 - 4 } & Tanh & 32.76 & 63.09 \\
\hline & Sigmoid & 33.20 & 69.71 \\
\cline { 2 - 4 } & ReLU & 58.00 & 66.90 \\
\cline { 2 - 4 } & Tanh & 59.80 & 69.50 \\
\hline & & & 6 \\
\hline
\end{tabular}

Fig. 13. F1 score Comparison Table between state-of-the-art method and our proposed model

\section{FUTURE WORK}

As observed in this paper, personality detection is a vital field that requires more research. Application of personality detection ranges from enhancing voice assistants (Alexa, Siri, Google Assistant, Cortana, etc.) to areas like forensics and health care. It is also used for hiring purposes and psychological studies.
Less biased word embeddings could be explored and the accuracy of the new model could be compared to the current model.

We have also observed the need of larger and more accurate datasets. Most ways of collecting data for this field include handing out questionnaires. Individuals may or may not answer the questions honestly and there must be a way to check the credibility of the responses.

\section{ACKNOWLEDGMENT}

We take this opportunity to thank Dr. Ruchika Malhotra for her valuable guidance throughout this research, from identifying the problem statement to presenting the research. We would also extend our thanks to Delhi Technological University for providing us with all the resources that were required to complete this research successfully.

\section{REFERENCES}

[1] Y. Mehta, N. Majumder, A. Gelbukh, and E. Cambria, "Recent trends in deep learning based personality detection," Artif. Intell. Rev., vol. 53, no. 4, pp. 2313-2339, 2020.

[2] K. C. Pramodh and Y. Vijayalata, "Automatic personality recognition of authors using big five factor model," 2016 IEEE Int. Conf. Adv. Comput. Appl. ICACA 2016, pp. 32-37, 2017.

[3] C. Ventura, D. Masip, and A. Lapedriza, "Interpreting CNN Models for Apparent Personality Trait Regression," in IEEE Computer Society Conference on Computer Vision and Pattern Recognition Workshops, 2017, vol. 2017-July, pp. 1705-1713.

[4] K. Gokul and S. Lalitha, "Personality Identification Using Auditory Nerve Modelling of Human Speech," 2018 Int. Conf. Adv. Comput. Commun. Informatics, pp. 1731-1737, 2018.

[5] E. F. M. Araújo, B. Simoski, and M. Klein, "Applying machine learning algorithms for deriving personality traits in social network," in Proceedings of the ACM Symposium on Applied Computing, 2018, pp. 346-349.

[6] A. Abyaa, M. Khalidi Idrissi, and S. Bennani, "Predicting the learner's personality from educational data using supervised learning," ACM Int. Conf. Proceeding Ser., pp. 1-7, 2018.

[7] Willy, E. B. Setiawan, and F. N. Nugraha, "Implementation of Decision Tree C4.5 for Big Five Personality Predictions with TFRF and TF-CHI2 on Social Media Twitter," 2019 Int. Conf. Comput. Control. Informatics its Appl. Emerg. Trends Big Data Artif. Intell. IC3INA 2019, pp. 114-119, 2019.

[8] T. Hong, X. Sun, F. Tian, and F. Ren, "Sentiment Classification And Personality Detection Via Galvanic Skin Response Based on Deep Learning Models," 2019 5th Int. Conf. Big Data Comput. Commun., pp. 313-317, 2019.

[9] I. B. Drexel, "Feature Engineering and Word Embedding Impacts for Automatic Personality Detection on Instant Message," in Proceedings of 2019 International Conference on Information Management and Technology, ICIMTech 2019, 2019, vol. 1, no. August, pp. 155-159.

[10] S. Gao, W. Li, L. J. Song, X. Zhang, M. Lin, and S. Lu, "PersonalitySensing: A Multi-View Multi-Task Learning Approach for Personality Detection based on Smartphone Usage," pp. 2862-2870, 2020.

[11] M. S. Salem, S. S. Ismail, and M. Aref, "Personality Traits for Twitter Users in the Egyptian Dialect Writing Dataset," Icsie, pp. 206-211, 2019.

[12] K. El-demerdash, R. A. El-khoribi, M. A. I. Shoman, and S. Abdou, "Psychological Human Traits Detection based on Universal Language Modeling," Egypt. Informatics J., no. xxxx, 2020.

[13] T. Yilmaz, A. Ergil, and B. İlgen, "Deep Learning-Based Document Modeling for Personality Detection from Turkish Texts," Adv. Intell. Syst. Comput., vol. 1069, pp. 729-736, 2020.

[14] M. A. Rahman, A. Al Faisal, T. Khanam, M. Amjad, and M. S. Siddik, "Personality Detection from Text using Convolutional Neural Network," 1st Int. Conf. Adv. Sci. Eng. Robot. Technol. 2019, ICASERT 2019, vol. 2019, no. Icasert, pp. 1-6, 2019. 
